\title{
ACTITUDES DE PADRES E HIJOS HACIA UN OCIO COMPARTIDO EN FAMILIA
}

\author{
ATTITUDES OF PARENTS AND CHILDREN TOWARDS SHARED FAMILY LEISURE \\ ATITUDES DE PAIS E FILHOS FACE A UM LAZER COMPARTILHADO \\ EM FAMÍLIA
}

\author{
Eva SANZ ARAZURI*, Magdalena SÁENZ DE JUBERA OCÓN* \& Rufino CANO** \\ *Universidad de la Rioja, ${ }^{* *}$ Universidad de Valladolid
}

PALABRAS CLAVE:

ocio

familia

hijos

joven

padres

percepción
Fecha de recepción: 18.111.2018

Fecha de revisión: 27.11.2018

Fecha de aceptación: 19.IV.2018

CONTACTO CON LOS AUTORES: Eva Sanz Arazuri. Universidad de La Rioja. Departamento de Ciencias de la Educación. Edificio Vives, Calle Luis de Ulloa s/n, 26004 Logroño. eva.sanz@unirioja.es

FINANCIACIÓN: El texto que presentamos se vincula al Proyecto de Investigación "De los tiempos educativos a los tiempos sociales: la cotidianidad familiar en la construcción del ocio físico-deportivo juvenil" (EDU2012-39080-C07-05), y configura los inicios del proyecto de I+D+| "Ocio y bienestar en clave intergeneracional: de la cotidianidad familiar a la innovación social en las redes abuelos-nietos" (EDU2017-85642-R); ambos cofinanciados en el marco del Plan Nacional I+D+l con cargo a dos ayudas del Ministerio de Economía y Competitividad, y por el Fondo Europeo de Desarrollo Regional (FEDER). 


\begin{tabular}{|c|c|}
\hline $\begin{array}{l}\text { KEY WORDS: } \\
\text { leisure } \\
\text { family } \\
\text { youth } \\
\text { son } \\
\text { parents } \\
\text { perception }\end{array}$ & $\begin{array}{l}\text { ABSTRACT: The family institution is a very important agent in the construction of its mem- } \\
\text { bers' entertainment, given its potential to make shared time become educational and val- } \\
\text { uable. It has been shown that shared family activities, including leisure, provide significant } \\
\text { benefits to those who practice it, which leads us to link personal and social growth, as well as } \\
\text { family welfare, with the time shared in the family. This study aims to analyze the attitude and } \\
\text { predisposition of Spanish students and their parents to family conciliation and shared family } \\
\text { leisure, considering variables such as the participation of each of its members in family activ- } \\
\text { ities, satisfaction about sharing free time, the amount of time they spend with the family, and } \\
\text { the degree of perceived pressure to spend more time together. A quantitative methodologi- } \\
\text { cal approach was carried out through the application of the Spanish adaptation of the FACES } \\
\text { IV questionnaire to a sample from Spain of } 1764 \text { students and } 839 \text { parents. This instrument } \\
\text { gathers information about cohesion and flexibility perceived within the family. The results } \\
\text { show that most Spanish students of post-compulsory secondary education and their parents } \\
\text { perceive that all the family members participate in family activities, showing a high degree of } \\
\text { satisfaction with shared leisure, which reveals a positive family atmosphere and very favorable } \\
\text { attitudes of parents and children toward the practice of leisure activities within the family. }\end{array}$ \\
\hline $\begin{array}{l}\text { PALAVRAS-CHAVE: } \\
\text { família } \\
\text { filhos } \\
\text { jovem } \\
\text { pais } \\
\text { perceção }\end{array}$ & $\begin{array}{l}\text { RESUMO: A instituição familiar é um agente de primeira ordem na construção do lazer dos } \\
\text { seus membros, dado o seu potencial para tornar o tempo compartilhado num tempo valioso } \\
\text { e educativo. Tem-se demonstrado que as atividades compartilhadas em família, incluindo as } \\
\text { atividades de lazer, proporcionam benefícios importantes para aqueles que as praticam, o } \\
\text { que permite vincular o crescimento pessoal e social, bem como o bem-estar da família, com } \\
\text { o tempo compartilhado em família. Este estudo tem como objetivo analisar a atitude e a } \\
\text { predisposição dos estudantes espanhóis e dos seus pais para a conciliação familiar e o lazer } \\
\text { compartilhado em família, tomando em consideração variáveis como a participação de cada } \\
\text { um de seus membros nas atividades familiares, a satisfação de compartilhar o tempo livre, a } \\
\text { quantidade de tempo que passam em família e o grau de pressão percebido para passarem } \\
\text { mais tempo juntos. Foi adotada uma abordagem metodológica quantitativa, desenvolvida } \\
\text { através da aplicação da adaptação espanhola do questionário FACES IV, para recolher in- } \\
\text { formação sobre a coesão e a flexibilidade percebida no seio da família, numa amostra que } \\
\text { incluiu } 1764 \text { estudantes de todo o território espanhol, e também } 839 \text { dos seus progenitores. } \\
\text { Os resultados evidenciaram que a maioria dos estudantes espanhóis do ensino secundário } \\
\text { pós-obrigatório e os seus pais percebem que todos os membros de suas famílias participam } \\
\text { em atividades familiares, mostrando um alto grau de satisfação em relação ao tempo livre que } \\
\text { compartilham, o que revela um ambiente familiar positivo e atitudes muito favoráveis de pais } \\
\text { e filhos face à prática de lazer familiar. }\end{array}$ \\
\hline
\end{tabular}

\section{Introducción}

La institución familiar es un espacio privilegiado para la transmisión de conocimientos fundamentales vinculados a la socialización, la convivencia, así como la adquisición y consolidación de actitudes, valores y hábitos derivados de las vivencias compartidas en el seno familiar (Elzo, 2004; Valdemoros, Ponce de León, Sanz \& Caride, 2014), por lo que se constituye en un agente de primer orden en la construcción del ocio de sus miembros, haciendo del tiempo compartido un tiempo potencialmente educativo (Muñoz \& Olmos, 2010).

El tiempo compartido en familia engloba tanto actividades cotidianas como no cotidianas, destacándose la parcela del tiempo libre, que se considera como un momento propio, exento de quehaceres, que se utiliza para realizar aquellas actividades deseadas y se caracteriza por las oportunidades que ofrece para aprender y compartir momentos, lo que implica predisposición para realizar algo que nos proporciona placer y bienestar (Lobo \& Menchén, 2004). En nuestra sociedad, el tiempo libre destinado a actividades de ocio está conquistando notabilidad en las últimas décadas, afianzándose en un derecho estimado por padres e hijos (Aristegui \& Silvestre, 2012) y en garante de desarrollo humano (Cuenca, Aguilar \& Ortega, 2010).

Diferentes líneas empíricas defienden que este tiempo compartido en familia, incluido el tiempo de ocio, reporta múltiples beneficios a quienes lo comparten, entre otros, en el bienestar (Maynard \& Harding, 2010; Offer, 2014), en el desarrollo intelectual, psicológico y social de los individuos (Craig \& Mullan, 2012; Shaw, 2008), en la salud, en su concepción holista (Grosso et al., 2011; Pinxten \& Lievens, 2014; Veenstra \& Patterson, 2012), así como su contribución positiva a la participación de los hijos en actividades de ocio futuras (Devos, 2006). Este tiempo compartido contribuye también a incrementar el grado de satisfacción que el joven tiene con su vida familiar (Agate, Zabriskie, Agate \& Poff, 2009; Barnes, Hoffman, Welte, Farrell \& Ditcheff, 2007; Offer, 2013; Zabriskie \& McCormick, 2001, 2003) de lo que se deriva que el hecho de compartir más tiempo en familia 
posibilita que progenitores e hijos disfruten de estos beneficios, lo que erige al tiempo compartido en familia en un indicador idóneo del bienestar familiar que depende, además de la cantidad de actividades compartidas, del tipo y la calidad de las mismas (Offer, 2014). Otros autores (Agate et al. 2009; Barnes et al. 2007; Crouter, Head, McHale \& Tucker, 2004; Offer, 2014; Zabriskie et al., 2003; Zaborskis, Zemaitiene, Borup, Kuntsche \& Moreno 2007) relacionan la percepción positiva de la calidad de vida de la familia con quienes intercambian más tiempo con sus miembros.

El ocio compartido en familia ha sido identificado, además, como uno de los principales factores de protección frente al desarrollo de comportamientos de riesgo (Barnes et al., 2007; Crouter et al., 2004; Wong, 2005), de tal modo que aquellos jóvenes que comparten más cantidad de este tiempo con sus familiares son los que tienen una menor probabilidad de desarrollar problemas comportamentales como pueden ser el consumo de alcohol, tabaco u otras drogas, argumento por el que las experiencias de ocio compartidas en familia pueden ser consideradas una estrategia con la que contribuir a reducir el ocio nocivo, que incluye aquellas experiencias que resultan perjudiciales para sus practicantes, así como para el entorno físico y social en el que viven (Cuenca, 2014).

Por otra parte, se descubren dificultades para la conciliación de los tiempos familiares, derivadas de las transformaciones culturales, ideológicas, sociales y económicas, como es el excesivo tiempo invertido en el trabajo, la estabilización de la mujer en el mercado laboral o la demora de la edad de emancipación, que afectan a la institución familiar, provocando numerosas dificultades para su organización y para la vida en comunidad. Estas cuestiones condicionan la cantidad y calidad del tiempo compartido entre padres e hijos, causando desazón y decepción en el seno familiar (Caballo, Gradaille \& Merelas, 2012; Fraguela, Lorenzo \& Varela, 2011).

Además, la sociedad del siglo XXI necesita reinventar la institución familiar y reconocer sus variadas configuraciones; así, la diversidad de estructuras y la convivencia de distintos tipos de familias podría ser una de los factores vinculados a las actividades compartidas entre padres e hijos, si bien existen estudios discrepantes sobre esta asociación, al igual que los hay respecto a la incidencia del número de hermanos que componen la unidad familiar (Zaborskis et al., 2007; Offer, 2014), lo que orienta a centrar el foco de atención en variables relacionadas con la dinámica familiar, dado que se constata que una buena organización, con interacciones positivas, reales y efectivas favorece los tiempos familiares compartidos (Pérez, Martínez, Mesa, Pérez \& Jiménez, 2009).

Lo mismo sucede con variables como el nivel educativo y/o económico familiar, el modelo de persona que ofrecen los progenitores a sus hijos o el estilo educativo de los padres, pues se encuentran investigaciones que las relacionan con actividades familiares y otras que las desligan (Valdemoros et al., 2014). Si bien, se descubre sintonía entre la literatura científica al señalarse como factor condicionante de un ocio familiar positivo el funcionamiento familiar, materializado en una cohesión y una flexibilidad familiar adecuadas (Hornberger, Zabriskie \& Freeman, 2010; Smith, Freeman \& Zabriskie, 2009), así como en una comunicación fluida entre progenitores y descendientes (Sharp, Graham \& Ridenour, 2006; Smith et al., 2009), que lleva a vivenciar prácticas conjuntas enriquecedoras, en pro del bienestar familiar (Agate, Zabriskie, Agate \& Poff, 2009). En este sentido se concluye que "la mejora de la organización familiar está vinculada con una mejora de la cohesión entre los miembros del núcleo familiar, así como en una mejora de la comunicación y la resolución cooperativa de problemas" (Orte, Ballester \& March, 2013, p. 21).

Asimismo, las dificultades para la conciliación familiar han derivado, en muchas ocasiones, en que muchos parientes que no conforman el núcleo familiar padres e hijos, acaben formando parte de la cotidianidad familiar, destacando los abuelos y abuelas. Ellos han adquirido una posición relevante dentro de la familia y una valiosa función social y educativa, y es que según datos de la encuesta europea SHARE (Survey of Health, Ageing and Retirement in Europe) un 36,8\% de los abuelos de 50 o más años afirman apoyar a la familia, fundamentalmente en el cuidado de sus nietos (Mari-Klose \& Escapa 2015), llevándoles a asumir en más ocasiones de las deseadas un rol de cuidadores, con el fin de dar respuesta a las necesidades de los padres, lejos de atender a sus necesidades como abuelos (González \& De la Fuente, 2007).

Por otra parte, los estudiantes de secundaria postobligatoria están en una etapa vital que se caracteriza por una ansia de libertad, por una necesidad de autonomía y de disfrutar de una vida independiente. Suelen pasar más tiempo en compañía de amigos, de pareja o, incluso, realizando pequeñas incursiones en el mundo laboral, generándose así cierto distanciamiento y desconexión familiar (Bell \& Bell, 2005, 2009) que suele traducirse en una reducción del tiempo compartido en familia. En esta línea, los hallazgos de Muñoz et al. (2010) constatan que los estudiantes de 
Educación Secundaria Obligatoria pasan más tiempo libre en familia en los primeros cursos de la etapa, descendiendo significativamente el tiempo compartido en los últimos cursos, si bien no difieren en cuanto al grado de satisfacción durante el tiempo libre que pasan familiarmente, manifestando todos estar bastante satisfechos. Esto lleva a considerar que conforme los alumnos avanzan en los niveles de escolarización, los tiempos compartidos tienden a disminuir, si bien suele perdurar el nivel de agrado con las actividades realizadas conjuntamente.

Estudios realizados por el Gabinete de Prospección Sociológica del Gobierno Vasco (2012, 2016) recogen la opinión de la ciudadanía en torno a la vida familiar. Sobre la frecuencia con la que ven a su familia, la mayoría de los encuestados se muestran muy satisfechos (49\%) o satisfechos (40\%), al igual que manifiestan un alto grado de satisfacción respecto a la frecuencia con la que comparten actividades (un $40 \%$ están muy satisfechos y un 38,5\% satisfechos). Respecto a este tiempo compartido entre todos los miembros del hogar, los estudios constatan que actividades cotidianas como charlar, desayunar, comer o cenar, así como ver la televisión se realizan mayoritariamente a diario o varias veces por semana; sin embargo, en lo referente a compartir las tareas de la casa hay más variabilidad, predominando que lo hacen casi nunca o nunca $(33 \%)$ seguida de diariamente (33\%) y de vez en cuando (19\%); el resto las realizan una o dos veces por semana (9\%) o los días festivos (3\%). Por otra parte, la frecuencia con que suelen hacer actividades no cotidianas como ver una película en casa, ir a algún museo, compartir un hobby, hacer deporte o jugar a las cartas suelen desarrollarse en un mayor porcentaje de vez en cuando o casi nunca.

\section{Justificación y objetivos}

Se ha puesto en evidencia que las actividades compartidas en familia reportan importantes beneficios a quienes las practican, lo que orienta a vincular el crecimiento personal y social, así como el bienestar familiar con quienes comparten más tiempo con los miembros de la familia. Si bien son numerosos los estudios realizados sobre jóvenes y tiempo libre, son escasos los que se centran en estudiantes de Educación Secundaria Postobligatoria fuera del entorno escolar, lo que motiva el interés de este estudio por tratar de responder algunos interrogantes: ¿Cómo perciben los estudiantes españoles y sus padres la participación de cada miembro de la familia en actividades familiares? ¿Opinan que el tiempo compartido es demasiado? ¿Existe presión familiar para incrementar ese tiempo? Además de los miembros que componen el núcleo familiar ¿̇se comparte el tiempo libre con otros parientes?

\section{Metodología}

El universo de este trabajo estuvo conformado por los estudiantes matriculados en Educación Secundaria Postobligatoria en algún centro educativo del Estado Español durante el curso 20132014 y por sus padres.

Conscientes de las dificultades para definir el universo de los padres y madres de estos estudiantes puesto que se puede encontrar una gran variabilidad en el número de padres vivos de cada uno de ellos, se decide tomar como unidad poblacional cada estudiante de Educación Secundaria Postobligatoria y uno de sus progenitores.

El tamaño de dicho universo fue identificado de los datos reflejados en la estadística de las Enseñanzas no universitarias de la Subdirección General de Estadística y Estudios del Ministerio de Educación, Cultura y Deporte. Un total de 1.055.532 alumnos se encontraban estudiando algún título de Educación Secundaria Postobligatoria durante el curso 2013-2014.

Dado que el universo objeto de estudio es finito, pero de un tamaño lo suficientemente amplio como para poder contemplar a cada una de las unidades poblacionales, la presente investigación se llevó a cabo a través de una selección muestral representativa. El tamaño muestral representativo para un error de muestreo de $\pm 2,3$ sigmas, un nivel de confianza del $95 \%$ y considerando el supuesto de que $\mathrm{p}=\mathrm{q}=0,5$, fue de 1764 estudiantes.

Se decidió emplear un muestreo probabilístico estratificado con afijación proporcional reflejando la representatividad de los estudiantes distribuidos por todo el estado español. Para ello, se atendió al conjunto de la geografía nacional identificando 6 áreas territoriales tomando como referencia las áreas delimitadas por el instituto de investigación de mercados Nielsen (tabla 1) y tratando de mantener una afijación proporcional en cada una de estas 6 áreas, se realizó un muestreo aleatorio simple de la población. Asimismo, dentro de cada área geográfica, se buscó una representatividad de cada una de las provincias, del sexo de los estudiantes, del tipo de enseñanzas de régimen general (bachillerato, ciclo formativo de grado medio y formación profesional básica), de la titularidad de los centros (públicos/privados), así como del número de aulas muestreadas en cada centro. 


\begin{tabular}{|l|l|}
\hline \multicolumn{2}{|c|}{ Tabla 1: Agrupación territorial para la muestra } \\
\hline Denominación/ Localización & \multicolumn{1}{c|}{ Comunidades y/o provincias } \\
\hline A1-Noreste & Cataluña, Aragón (excepto Teruel) y Baleares \\
\hline A2-Levante & Comunidad Valenciana, Murcia y Albacete \\
\hline A3-Sur & Andalucía, Islas Canarias, Ceuta y Melilla \\
\hline A4-Centro & $\begin{array}{l}\text { Madrid, Castilla-La Mancha (excepto Albacete), Castilla-León (excepto León, Palencia y } \\
\text { Burgos), Cáceres y Teruel }\end{array}$ \\
\hline A5-Noroeste & Galicia, Asturias y León \\
\hline A6-Norte & Cantabria, País Vasco, La Rioja, Navarra, Burgos y Palencia \\
\hline Fuente: elaboración propia. & \\
\hline
\end{tabular}

De los 1764 estudiantes participantes en la muestra, la media de edad fue de 17,60 $\pm 1,60$ años, el $50,1 \%$ fueron mujeres $(n=885)$ y el $49,9 \%$ hombres $(n=879)$. El $67 \%$ fueron estudiantes de Bachillerato, el 32,7\% alumnado de ciclo formativo de grado medio y el 10,3\% discentes de formación profesional básica. La gran mayoría de los jóvenes españoles de Educación Secundaria Postobligatoria durante el curso 2013-2014 poseían la nacionalidad española (89,6\%; $n=1581)$.

En total se consiguió recopilar la información de los padres del $47,56 \%$ de los estudiantes participantes en la muestra $(n=839)$. La media de edad de estos padres fue de 49,45 \pm 1,60 años, el $66,6 \%$ de los progenitores que respondieron al instrumento fueron mujeres $(n=559)$ y el $33,3 \%$ hombres $(n=280)$. La gran mayoría poseían la nacionalidad española $(86,29 \% ; n=724)$.

Se establecen 4 variables instrumentales definidas por Olson (2008) que han permitido a este estudio analizar la actitud y predisposición de los estudiantes de secundaria postobligatoria y de sus padres hacia la conciliación familiar y un ocio compartido en familia y 1 variable que identifica el rol familiar del encuestado.

- Participación de cada miembro de la familia en actividades familiares: Esta variable, a través de una escala tipo Likert de 5 puntos, identifica el grado en que estudiantes y padres perciben que cada miembro de su familia participa en las actividades familiares. Las categorías definidas son:

1: Totalmente en desacuerdo

2: Generalmente en desacuerdo

3: Indeciso

4: Generalmente de acuerdo

5: Totalmente de acuerdo
- Gusto por pasar tiempo libre con sus familiares: Recoge el grado en que estudiantes y padres perciben que les gusta pasar tiempo con sus familiares a través de una escala tipo Likert de 5 niveles:

1: Totalmente en desacuerdo

2: Generalmente en desacuerdo

3: Indeciso

4: Generalmente de acuerdo

5: Totalmente de acuerdo

- Percepción del tiempo familiar compartido:

A través de esta variable se registra si estudiantes y padres consideran que el tiempo que pasan en familia es escaso o excesivo. Para ello, se emplea una escala tipo Likert de 5 puntos en la que los participantes han de señalar su grado de acuerdo o desacuerdo con la siguiente afirmación "pasamos demasiado tiempo juntos" donde significa:

1: Totalmente en desacuerdo

2: Generalmente en desacuerdo

3: Indeciso

4: Generalmente de acuerdo

5: Totalmente de acuerdo

- Grado de presión percibida para pasar más tiempo con su familia: Esta variable, a través de una escala tipo Likert de 5 puntos, examina si estudiantes y padres se sienten presionados para pasar más tiempo con su familia. Las categorías contempladas son:

1: Totalmente en desacuerdo

2: Generalmente en desacuerdo

3: Indeciso

4: Generalmente de acuerdo

5: Totalmente de acuerdo

- Rol familiar adoptado: variable dicotómica con dos categorías, hijo (estudiante) o padre. 
Los datos de estas variables se consiguieron mediante las respuestas de los estudiantes y de uno de sus progenitores a la adaptación española del cuestionario FACES IV (Rivero, Martínez-Pampliega y Olson, 2010), que recoge información sobre la cohesión y la flexibilidad percibida en el seno familiar.

La aplicación de este instrumento se realizó como parte de recogida de información de una investigación más extensa y compleja, “De los tiempos educativos a los tiempos sociales: la construcción cotidiana de la condición juvenil en una sociedad de redes. Problemáticas y alternativas pedagógico-sociales", que profundizó sobre características del alumno, vida en el centro escolar, vida familiar, tiempo libre, salud y calidad de vida, estudios y mercado laboral en el futuro, emprendimiento y funcionamiento familiar.

Antes de la aplicación definitiva del instrumento, se llevó a cabo una prueba piloto en la que participaron 248 estudiantes de ocho Comunidades Autónomas y de diferentes tipos de estudios de Educación Secundaria Postobligatoria. Asimismo, se contó con el juicio de catorce expertos investigadores de siete universidades españolas diferentes.

Para la aplicación definitiva del instrumento se empleó la técnica de conglomerados, seleccionando al azar centros educativos de cada una de las 6 áreas territoriales teniendo en cuenta dos criterios de inclusión: la necesaria participación de un centro rural por área territorial y mantener una proporción de un centro de régimen privado-concertado por cada tres de titularidad pública. En cada uno de los centros seleccionados se celebró una sesión única para la cumplimentación del cuestionario por parte del número de estudiantes necesarios para cubrir la cuota muestral definida. Antes de su aplicación, se solicitó permiso al Director General de Educación de cada Comunidad Autónoma y se contacta con los directores de los centros educativos seleccionados para, tras informarles sobre los objetivos del estudio, solicitar la posible participación de algunos de sus alumnos en la cumplimentación del cuestionario. Dos investigadores debidamente formados acudieron personalmente a cada centro, para dirigir, ayudar y coordinar la cumplimentación del cuestionario aumentando la fiabilidad y reduciendo la mortalidad experimental.

El análisis y tratamiento de los datos se presenta en tres niveles. En una primera fase se muestra un análisis de frecuencias de cada una de las variables de estudio, lo que permite reflejar tanto la actitud y predisposición de los estudiantes españoles de Secundaria Postobligatoria como la de sus padres, hacia la conciliación familiar y un ocio compartido en familia. Posteriormente, se empleó el coeficiente de correlación de Pearson y se determinó que para valores de $r$ menores o iguales a ,200 la correlación era muy débil, entre ,200 y ,400 débiles, entre ,400 y ,600 moderadas, entre, 600 y, 800 fuertes y muy fuertes para valores entre ,800 y 1. Este análisis estadístico permitió identificar para cada una de las variables estudiadas si el crecimiento de la percepción de los estudiantes guardaba una relación directa o inversa con la percepción de los padres. Finalmente, se procedió a calcular la diferencia entre los valores perceptivos señalados por el estudiante y por su padre en cada unidad muestral contrastando si la media difería significativamente con el fin de poder determinar si los estudiantes o sus padres muestran una actitud y predisposición significativamente superior hacia la conciliación familiar y un ocio compartido en familia. Para ello se empleó la comparación de medias mediante la prueba $t$ para dos muestras relacionadas.

Para todo el estudio se fijó como nivel de significatividad un valor de $\mathrm{p}$ inferior a 0,05.

\section{Resultados}

El 60,5\% de los estudiantes españoles de Educación Secundaria Postobligatoria y el $78,4 \%$ de sus padres perciben que cada miembro de su familia participa en actividades familiares; un $23,4 \%$ de jóvenes y un $13 \%$ de progenitores se muestran indecisos mientras que un $16,2 \%$ y un $8,6 \%$ respectivamente no perciben una participación de todos los miembros de su familia en actividades conjuntas (figura 1).

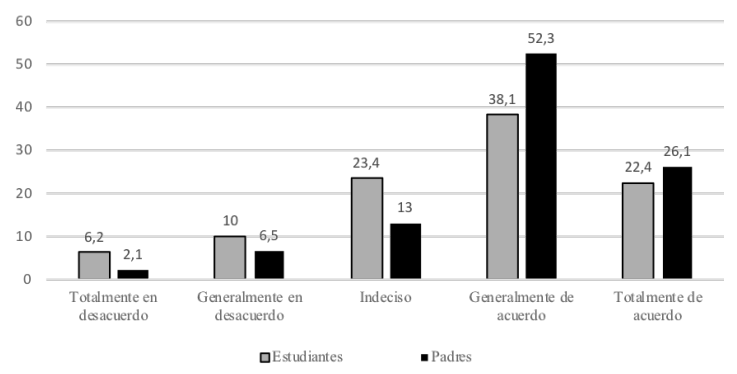

Figura 1. Percepción sobre la participación de cada miembro de la familia en actividades familiares

El análisis de correlación muestra una relación lineal significativa aunque leve, entre las percepciones de los estudiantes y las de sus padres ( $r$ de Pearson=,136 p=,000), Valores altos de acuerdo de los estudiantes se corresponden con valores altos de los padres. 


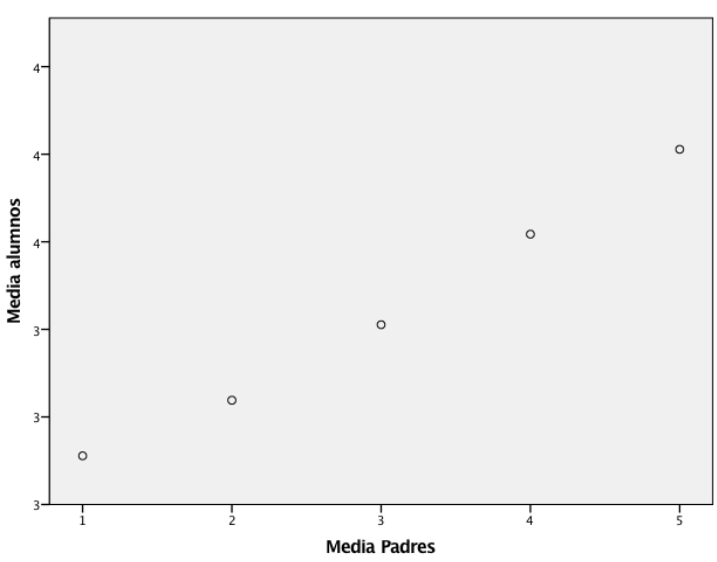

Figura 2. Dispersión lineal entre la percepción de padres e hijos ante la afirmación "Cada miembro de la familia participa en las actividades familiares"

Por su parte, la comparación de medias identifica una diferencia significativa $(p=, 000)$, revelando que los padres perciben en mayor medida una participación de todos los miembros de su familia en las actividades familiares ( $\overline{\mathrm{x}}$ estudiantes $=3,6 \pm$ 1,121 vs $\bar{x}$ padres $=4,1 \pm 1,687$ ).

El $57,2 \%$ de los estudiantes y el $81,7 \%$ de sus padres perciben que a los miembros de su familia les gusta pasar tiempo libre juntos; un $25,5 \%$ de jóvenes y un $10,9 \%$ de progenitores se muestran indecisos mientras que un $17,3 \%$ y un $7,3 \%$ respectivamente creen que a los miembros de su familia no les gusta compartir tiempo libre (figura 3).

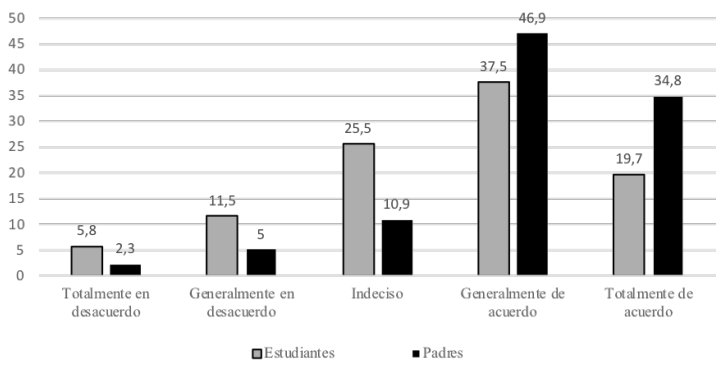

Figura 3. Percepción sobre si les gusta pasar su tiempo libre con sus familiares

El análisis de correlación muestra una relación lineal significativa aunque leve entre las percepciones de los estudiantes y las de sus padres ( $r$ de Pearson=,149 p=,000), De nuevo, valores altos de acuerdo de los estudiantes se corresponden con valores altos de los padres.

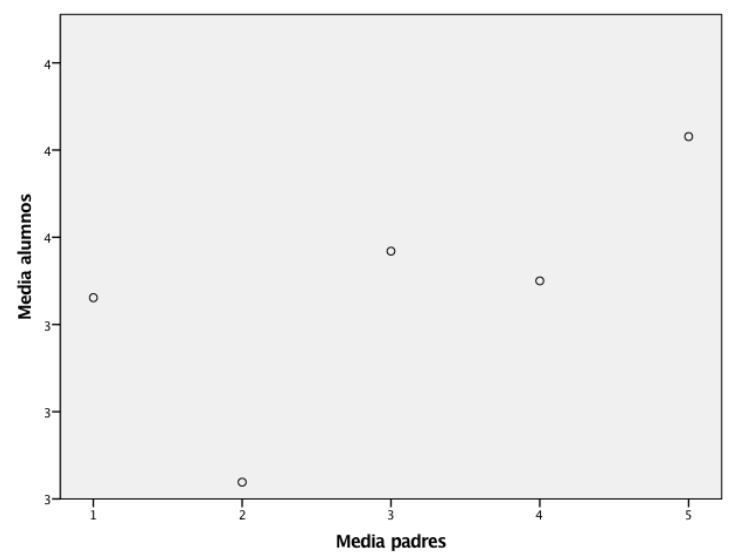

Figura 4. Dispersión lineal entre la percepción de padres e hijos ante la afirmación "Nos gusta pasar algo de nuestro tiempo libre con los otros miembros de la familia"

Por su parte, la comparación de medias identifica una diferencia significativa $(p=, 000)$, revelando que los padres perciben un mayor deseo por parte de los miembros de su familia por compartir tiempo libre ( $\overline{\mathrm{x}}$ estudiantes=3,5 $\pm 1,105$ vs $\overline{\mathrm{x}}$ padres $=4,1 \pm 0,930$ ).

En cuanto a la percepción que muestran hijos y padres respecto al tiempo que pasan juntos, el $20,2 \%$ de los estudiantes y el $22,9 \%$ de sus padres perciben que pasan demasiado tiempo juntos; un $30 \%$ de jóvenes y un $17,2 \%$ de padres se muestran indecisos mientras que un $49,7 \%$ y un $59,8 \%$ no están de acuerdo con esa afirmación (figura 5).

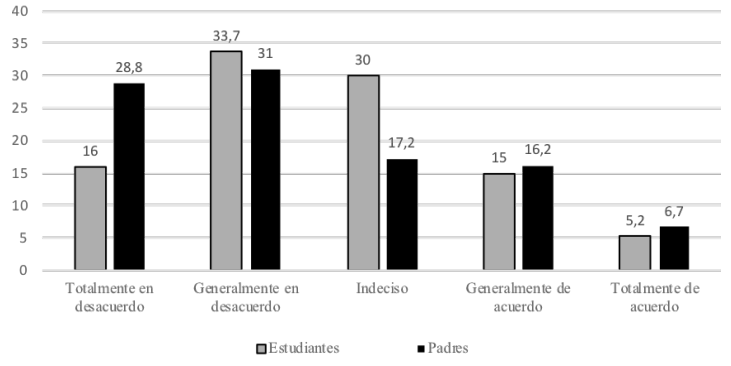

Figura 5. Grado de acuerdo con la afirmación "pasamos demasiado tiempo juntos"

El análisis de correlación muestra una relación lineal significativa aunque leve entre las percepciones de los estudiantes y las de sus padres ( $r$ de Pearson=,177 $p=, 000$ ), En esta variable se vuelven a repetir la correspondencia de valores altos de acuerdo de los estudiantes con valores altos de los padres. 


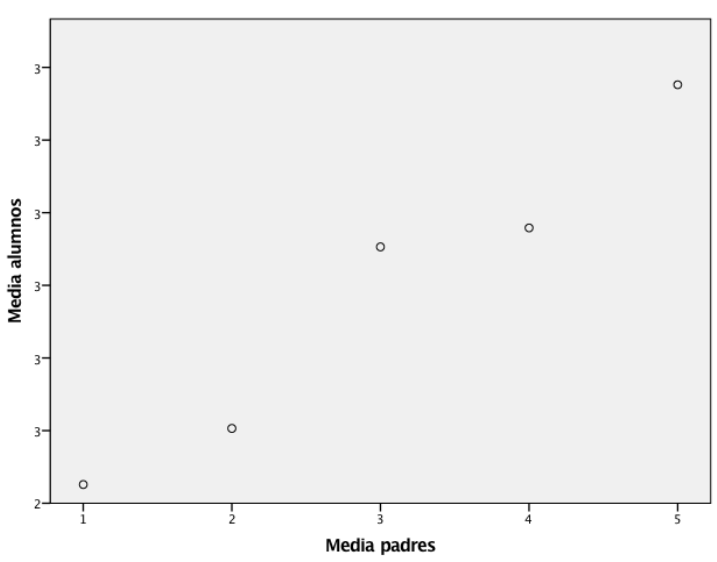

Figura 6. Dispersión lineal entre la percepción de padres e hijos ante la afirmación "pasamos demasiado tiempo juntos"

Por su parte, la comparación de medias no identifica una diferencia significativa $(p=, 177)$, revelando que padres e hijos muestran el mismo desacuerdo ante la idea de que pasan demasiado tiempo juntos ( $\overline{\mathrm{x}}$ estudiantes $=2,6 \pm 1,084$ vs $\overline{\mathrm{x}}$ padres $=2,4 \pm 1,280$ ).

Respecto a la presión que sienten por los miembros de su familia para pasar tiempo juntos, tan solo el 9,8\% de los jóvenes y el $8 \%$ de los progenitores perciben sentirse presionados; un $16,6 \% \%$ de jóvenes y un $8,4 \%$ de padres se muestran indecisos mientras que un $73,7 \%$ y un $83,5 \%$ no se sienten presionados (figura 7).

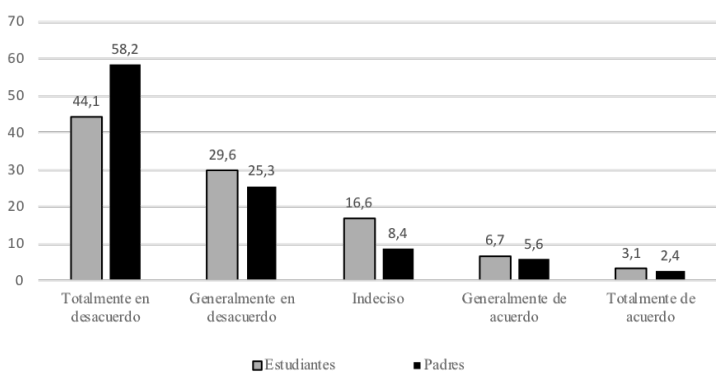

Figura 7. Percepción de sentirse presionado para pasar más tiempo con los miembros de su familia

El análisis de correlación también en este caso muestra una relación lineal significativa aunque leve entre las percepciones de los estudiantes y las de sus padres ( $r$ de Pearson=,153 $p=, 000$ ). En esta variable se vuelven a repetir la correspondencia de valores altos de acuerdo de los estudiantes con valores altos de los padres.

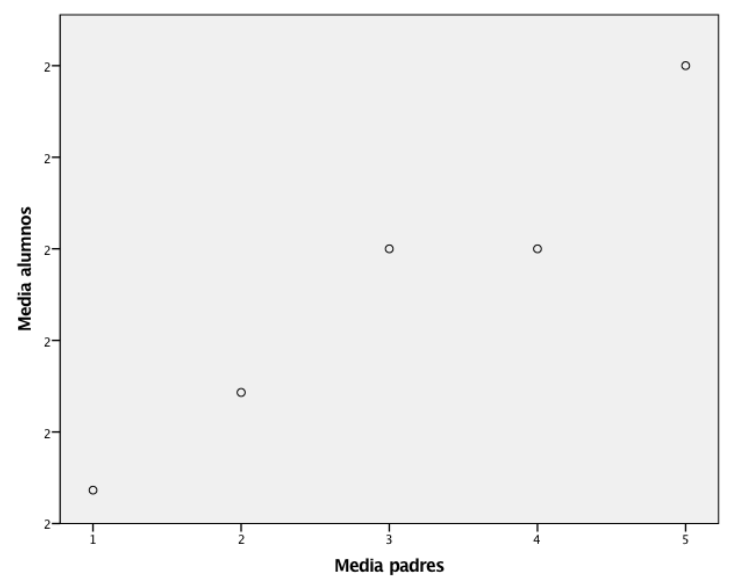

Figura 8. Dispersión lineal entre la percepción de padres e hijos ante la afirmación "Nos sentimos presionados para pasar la mayor parte del tiempo juntos"

Por su parte, la comparación de medias no identifica una diferencia significativa $(p=, 741)$, revelando que padres e hijos se muestran poco presionados por sus familias para pasar más tiempo juntos ( $\overline{\mathrm{x}}$ estudiantes $=1,95 \pm 1,071$ vs $\overline{\mathrm{x}}$ padres $=1,69$ $\pm 1,008)$.

\section{Discusión y conclusiones}

El presente estudio revela que la mayor parte de los estudiantes españoles de educación secundaria postobligatoria, así como sus padres, perciben que todos los miembros de sus familias participan en las actividades familiares, destacando el grado de acuerdo entre la impresión de hijos y padres al respecto, aunque la de los padres es significativamente más positiva. Estos resultados concuerdan con los ofrecidos por los estudios del Gabinete de Prospección Sociológica del Gobierno Vasco (2012-2016) que reflejan un alto grado de satisfacción (cercano al $80 \%$ ) respecto a la frecuencia con que participan en actividades cotidianas como no cotidianas todos los miembros de la familia, si bien las poblaciones objeto de estudio son distintas. También Buxarrais y Escudero (2014) hacen percibir esta situación esperanzadora, al corroborar que la distribución de las tareas familiares entre todos sus miembros favorece el equilibrio entre la conciliación familiar, laboral y estudiantil, facilita el desarrollo personal de cada miembro de la familia, así como la convivencia entre padres e hijos y la mejora la salud familiar, generando paralelamente un incremento de las oportunidades para la promoción de experiencias de ocio en familia.

Estudios previos como el de Ponce de León, Sanz y Valdemoros (2015) revelan que la mayoría de los estudiantes de Educación Secundaria Postobligatoria desean dedicar tiempo libre a estar con 
su familia (3 de cada 4), lo que se situa en sintonía con el presente trabajo que afirma no solo que los jóvenes objeto de estudio constatan que su familia desea compartir parte de su tiempo libre (6 de cada 10), sino que además, aporta que es muy alta la proporción de padres que desean compartir su tiempo libre con sus hijos (8 de cada 10).

Estos resultados, también concuerdan con los hallazgos obtenidos por Muñoz et al. (2010), quienes afirman que los jóvenes se muestran bastante satisfechos respecto al tiempo libre que pasan familiarmente, si bien concluye que la relación entre padres e hijos cambia tanto de forma cuantitativa, en cuanto a que los jóvenes pasan menos tiempo en el hogar, como cualitativa, en referencia a que comparten más sus temas personales con los iguales, si bien siguen necesitando a sus progenitores para conformar sus valores.

Además, con este trabajo se refleja una asociación lineal entre padres e hijos, pues se verifica que cuanto más perciben los padres un deseo familiar por compartir su tiempo libre, mayor es también el deseo percibido por los hijos.

Otra aportación relevante es que tanto los estudiantes como los padres no consideran que el tiempo que pasan juntos sea excesivo, lo que apoya favorablemente el potencial éxito de la promoción de actividades de ocio compartidas en familia reportando bienestar a quiénes las vivencian (Maynard \& Harding, 2010; Offer, 2014), desarrollo intelectual, psicológico y social a cada uno de sus miembros (Craig \& Mullan, 2012; Shaw,
2008) y mejora de la salud, en su concepción holista (Grosso et al., 2011; Pinxten \& Lievens, 2014; Veenstra \& Patterson, 2012).

La presente investigación también descubre una ausencia de presión, tanto en los jóvenes que estudian educación secundaria postobligatoria, como en sus padres, ante el hecho de pasar la mayor parte del tiempo libre con su familia, lo que parece apuntar a una flexibilidad familiar adecuada (Hornberger, Zabriskie \& Freeman, 2010; Smith, Freeman \& Zabriskie, 2009) que unida al agrado que estas actividades compartidas les aportan (Muñoz et al. 2010) favorece una actitud abierta hacia el incremento de momentos familiares más allá de los que ya experimentan.

En conclusión, los resultados de este estudio muestran un ambiente familiar y unas actitudes de padres e hijos muy favorables para la práctica de ocio en familia. Sin embargo, tal y como nos muestran estudios como Berntsson \& Rigsberg (2014), son escasos los jóvenes que reconocen compartir experiencias de ocio con quienes mantienen un parentesco directo, más allá de ver la televisión o ir de compras. Debido a la escasez de investigación al respecto, sería de gran interés para la comunidad científica comprobar qué factores impiden o dificultan estas actividades compartidas en familia con el fin de aportar claves para su promoción eficiente, su fomento eficaz y su fácil acceso, favoreciendo el interés de los jóvenes españoles y sus padres por un ocio compartido en familia.

\section{Referencias bibliográficas}

Agate, J. R., Zabriskie, R. B., Agate, S. T., \& Poff, R. (2009). Family Leisure Satisfaction and Satisfaction with Family Life. Journal of Leisure Research, 47(2), 205-223.

Aristegui, I., \& Silvestre, M. (2012). El ocio como valor en la sociedad actual. Arbor, 188, 283-291. http://dx.doi.org/10.3989/ arbor.2012.754n2001

Barnes, G., Hoffman, J., Welte, J., Farrell, M., \& Dintcheff, B. (2007). Adolescents' Time Use: Effects on Substance Use, Delinquency and Sexual Activity. Journal of Youth and Adolescence, 36(1), 697-710. doi: http://dx.doi.org/10.1007/ s10964-006-9075-0

Bell, L., \& Bell, D. (2005). Family dynamics in adolescence affect midlife well-being. Journal of Family Psychology, 19(1), 198-207. doi: http://dx.doi.org/10.1037/0893-3200.19.2.198

Bell, L., \& Bell, D. (2009). Effects of family connection and family individuation. Attachment \& Human Development, $11(5)$, 471-490. doi: http://dx.doi.org/10.1080/14616730903132263

Berntsson, L. T., \& Ringsberg, K. C. (2014). Swedish parents' activities together with their children and children's health: A study of children aged 2-17 years. Scandinavian Journal of Public Health, 42(15), 41-51. doi:10.1177/1403494814544901

Buxarrais M. R., \& Escudero, A. (2014). Ocio en familia: una estrategia para promover el valor de la corresponsabilidad en la familia. En M.R. Buxarrais y M. Burguet (Ed.), La conciliación familiar, laboral, social y personal: una cuestión ética (pp. 105-128). Barcelona, España: Publicacions i edicions de la Universitat de Barcelona

Caballo, M., B., Gradaílle, R. \& Merelas, T. (2012). Servicios socioeducativos y corresponsabilidad en la conciliación de los tiempos familiares: situación de la infancia en la Galicia urbana. Pedagogía Social. Revista Interuniversitaria, 20,179-202. doi: 10.7179/PSRI 
Craig, L., \& Mullan, K. (2012). Shared Parent-Child Leisure Time in Four Countries. Leisure Studies, 31(2), 211-229. doi:10. 1080/02614367.2011.573570

Crouter, A. C., Head, M. R., McHale, S.M., \& Tucker, C. J. (2004). Family time and the psychosocial adjustment of adolescent siblings and their parents. Journal of Marriage and Family, 66(1), 147-162. doi: http://dx.doi. org/10.1111/j.0022-2445.2004.00010.x-i1

Cuenca, M., Aguilar, E., \& Ortega, C. (2010). Ocio para innovar. Bilbao: Universidad de Deusto, Documentos de estudios de ocio, 42

Cuenca, M. (2014). Ocio valioso. Bilbao: Universidad de Deusto

Devos, F. (2006). Jóvenes pero alcanzables. Técnicas de marketing para acercar la cultura a los jóvenes. Madrid: Fundación Autor.

Elzo, J. (2004). Tipología y socialización de las familias españolas. Arbor, 178(702), 205-229. http://dx.doi.org/10.3989/ arbor.2004.1702.566

Fraguela, R., Lorenzo, J. J., \& Varela, L. (2011). Escuela, familias y ocio en la conciliación de los tiempos cotidianos en la infancia. Revista de Investigación Educativa, 29(2), 429-446. Recuperado de: http://goo.gl/tnjeFW

Gabinete de prospección sociológica del Gobierno Vasco (2012). La familia en la CAPV. Gobierno Vasco.

Gabinete de prospección sociológica del Gobierno Vasco (2017). La familia. Gobierno Vasco.

González, J., \& De la Fuente, R. (2007). Intergenerational grand-parent/grandchild relations: the socioeducational role of grand-parents. Educational Gerontology, 34, 67-88. doi: 10.1080/03601270701763993

Grosso, G., Marventano, S., Nolfo, F., Rametta, S., Bandini, L., Ferranti, R.,... Mistretta, A. (2013). Personal eating, lifestyle, and family-related behaviours correlate with fruit and vegetable consumption in adolescents living in Sicily, southern Italy. International Journal for Vitamin and Nutrition Research, 83(6), 355-366. doi: http://dx.doi. org/10.1024/0300-9831/a000177

Hornberger, L. B., Zabriskie, R. B., \& Freeman, P. (2010). Contributions of Family Leisure to Family Functioning Among Single-Parent Families. Leisure Sciences, 32 (2), 143-161, http://dx.doi.org/10.1080/01490400903547153

Lobo, J. L., \& Menchén, F. (2004). Libertad y responsabilidad en el tiempo libre. Estrategias y pautas para padres y educadores. Madrid: Pirámide.

Mari-Klose, P. y Escapa Solanas, S. (2015). Solidaridad intergeneracional en época de crisis: ¿̇mito o realidad? Panorama Social, 22(2), 61-78.

Maynard, M. J., \& Harding, S. (2010). Ethnic differences in psychological well-being in adolescence in the context of time spent in family activities. Social Psychiatry and Psychiatric Epidemiology, 45(1), 115-123. doi: https://doi.org/10.1007/ s00127-009-0047-z

Muñoz, J. M., \& Olmos, S. (2010) Adolescencia, tiempo libre y educación. Un estudio con alumnos de la ESO. Educación $X X_{1}, 13(2), 139-162$.

Offer, S. (2014). Time with children and employed parents' emotional well-being. Social science research, 47, 192-203. doi: https://doi.org/10.1016/j.ssresearch.2014.05.003

Olson, D. H. (2008). FACES. IV Manual. Minneapolis (MN): Life Innovations.

Orte, C., Ballester, LI., \& March, M. (2013). El enfoque de la competencia familiar, una experiencia de trabajo socioeducativo con familias. Pedagogía Social, Revista Interuniversitaria, 21, 13-37.

Pérez Milena, A., Martínez Fernández, M. L., Mesa, I., Pérez Milena, R., Leal, F. J., \& Jiménez Pulido, I. (2009). Cambios en la estructura y en la función familiar del adolescente en la última década (1997-2007). Atención primaria, 47 (9), 479-486. http://dx.doi.org/10.1016/j.aprim.2009.03.015

Pinxten, W., \& Lievens, J. (2014). The importance of economic, social and cultural capital in understanding health inequalities: using a Bourdieu-based approach in research on physical and mental health perceptions. Sociol Health \& Illn. 36(7), 1095-110. doi: http://dx.doi.org/10.1111/1467-9566.12154

Rivero, N., Martínez-Pampliega, A., \& Olson, D. (2010). Spanish Adaptation of the FACES IV Questionnaire. Psychometric Characteristics. The Family Journal, 18, 288-296.

Sharp, E. H., Caldwell, L. L., Graham, J. W., \& Ridenour, T. A. (2006). Individual motivation and parental influence on adolescents' experiences of interest in free time: a longitudinal examination. Journal of youth and adolescence, 35(3), 359-372.

Shaw, S. (2008). Family Leisure and Changing Ideologies of Parenthood. Sociology Compass, 2 (2), 688-703. doi: https:// doi.org/10.1111/j.1751-9020.2007.00076.x

Smith, K. M., Freeman, P. A., \& Zabriskie, R. B. (2009). An Examination of Family Communication Within the Core and Balance Model of Family Leisure Functioning. Family Relations, 58 (7), 79-90.

Valdemoros, M. A., Ponce de León A., Sanz, E., \& Caride, J. A. (2014). La influencia de la familia en el ocio físico-deportivo juvenil: Nuevas Perspectivas para la reflexión y la acción. Arbor, 190(770): a192. doi: https://doi.org/10.3989/ arbor.2014.770n6013 
Veenstra, G., \& Patterson, A. C. (2012) Capital relations: mediating and moderating effects of cultural, economic, and social capitals on mortality in Alameda County, California, International Journal of Health Services, 42(2), 277-291. doi: http://dx.doi.org/10.1111/1467-9566.12105

Wong, S. K. (2005). The effects of adolescent activities on delinquency: A differential involvement approach. Journal of Youth and Adolescence, 34(4), 321-333. doi:10.1007/s10964-005-5755-4

Zaborskis, A., Zemaitiene, N., Borup, I., Kuntsche, E., \& Moreno, C. (2007). Family joint activities in a cross-national perspective. BMC Public Health, 7(94), 1-14. doi:10.1186/1471-2458-7-94

Zabriskie, R. B., \& McCormick, B. P. (2001). The Influences of Family Leisure Patterns on Perceptions of Family Functioning. Family Relations, 5O(3), 281-289. doi: http://dx.doi.org/10.111/j.1741-3729.2001.00281.x

Zabriskie, R. B., \& McCormick, B. P. (2003). Parent and child perspectives of family leisure involvement and satisfaction with family life. Journal of Leisure Research, 35(2), 163-189. Recuperado de: http://goo.gl/4acrxf

\section{CÓMO CITAR ESTE ARTÍCULO}

Sanz, E., Sáenz de Jubera, M., \& Cano, R. (2018). Actitudes de padres e hijos hacia un ocio compartido en familia. Pedagogía Social. Revista Interuniversitaria, 32 59-70. DOI:10.7179/PSRI_2018.32.05

\section{DIRECCIÓN COMPLETA DE LOS AUTORES}

Eva Sanz Arazuri. Universidad de La Rioja. Departamento de Ciencias de la Educación. Edificio Vives, Calle Luis de Ulloa s/n, 26004 Logroño. eva.sanz@unirioja.es

Magdalena Sáenz de Jubera Ocón. Universidad de La Rioja. Departamento de Ciencias de la Educación. Edificio Vives, Calle Luis de Ulloa s/n, 26004 Logroño. magdalena.saenz-de-jubera@ unirioja.es

Rufino Cano. Universidad de Valladolid, Facultad de Educación y Trabajo Social. Departamento de Pedagogía. MIDE. Campus Miguel Delibes. Paseo de Belén s/n, 47011 Valladolid. rcanogonzalez@gmail.com

\section{PERFIL ACADÉMICO}

Eva Sanz Arazuri. Profesora Titular de Universidad en el área Didáctica de la Expresión Corporal de la Universidad de La Rioja. Doctora en Educación Física por la Universidad de Zaragoza. Desde el año 2000 es investigadora en el campo del ocio, la juventud, la familia y los comportamientos físico-deportivos, como miembro del Grupo de investigación AFYDO de la Universidad de La Rioja. En la actualidad es co-investigadora principal en un proyecto nacional de $1+D+\mid$ (2018-2020), financiado por el Ministerio de Economía y Competitividad en la convocatoria de proyectos de investigación fundamental no orientada (Ref. EDU2017-85642-R) que persigue analizar el ocio y bienestar familiar en clave intergeneracional prestando especial atención en las redes abuelos-nietos. 
Magdalena Sáenz de Jubera Ocón. Profesora Contratada Interina en el área Didáctica de la Organización Escolar de la Universidad de La Rioja. Doctora por la Universidad de La Rioja. Es investigadora una línea de investigación relacionada campo del ocio, los valores, el diagnóstico y la orientación profesional, y el estudio de propuestas de Educación Inclusiva para los centros educativos. Miembro del Grupo de investigación AFYDO de la Universidad de La Rioja. En la actualidad es investigadora del proyecto nacional de l+D+l (2018-2020), financiado por el Ministerio de Economía y Competitividad en la convocatoria de proyectos de investigación fundamental no orientada (Ref. EDU2017-85642-R) que persigue analizar el ocio y bienestar familiar en clave intergeneracional prestando especial atención en las redes abuelos-nietos.

Rufino Cano. Profesor Titular de Universidad en el área MIDE de la Universidad de Valladolid. Doctor en Filosofía y Ciencias de la Educación. Desde 2013 colabora con el grupo de investigación AFYDO (Universidad de La Rioja). Es miembro del Grupo de Investigación, Red Colombiana de Investigación en Entornos Virtuales y a Distancia (RECIEVAD). Bogotá (Colombia). En la actualidad es investigador del proyecto nacional de I+D+l (2018-2020), financiado por el Ministerio de Economía y Competitividad en la convocatoria de proyectos de investigación fundamental no orientada (Ref. EDU2017-85642-R) que persigue analizar el ocio y bienestar familiar en clave intergeneracional prestando especial atención en las redes abuelos-nietos. 